\title{
MHD Kamchatnov-Hopf soliton in the model of primordial solar nebula
}

\author{
Vladimir V. Salmin \\ Siberian Federal University, \\ Svobodny ave., 79, Krasnoyarsk, 660041, Russia \\ email: vsalmin@gmail.com
}

\begin{abstract}
Stereographic projection of Hopf field on the 3-sphere into Euclidean 3-space is used as a model of 3D steady flow of ideal compressible fluid in MHD. In such case, flow lines are Villarceau circles lying on tori corresponding to the levels of Bernoulli function. Existence of an optimal torus with minimal relative surface free energy is shown. Beat of oscillations with wave numbers corresponding to structural radii of optimal torus leads to scaling of optimal tori. Spatial intersection of homothetic tori within one torus result in formation of cluster with the size depending on scaling factor. Optimal tori are considered as precursors of planetary orbits.
\end{abstract}

Keywords. MHD, solar system: formation

\section{Introduction}

Solution of the equations of ideal magnetohydrodynamics describes a localized topological soliton with use of Hopf mapping shown by Kamchatnov (1982). Example of introducing the Euler's potential into a topological MHD soliton which has non-trivial helicity called MHD Kamchatnov-Hopf soliton was described by Semenov et al. (2001). Hopf field on $S^{3}$ has minimal energy among all the fields diffeomorphic to it (Arnold \& Khesin 1998). Stability of Hopf field on $S^{3}$ has been proved (Gil-Merdano \& LlinaresFuster 2001, Yampolsky 2003). Hopf fibration of $S^{3}$ with stereographic projection induces the toroidal coordinates on $E^{3}$ (Gibbons 2006).

\section{Overview}

On a torus $\nu=\operatorname{arcsinh}(k) / k$ contravariant metrical tensor in toroidal coordinates is $\left.g^{i j}\right|_{T}=\delta_{i}^{j}\left(\sqrt{k^{2}+1}-\cos (k \alpha)\right)^{2} / R^{2} k^{2}$. For steady flow of ideal compressible fluid, stress tensor is determined by metrical tensor only $\left.p^{i j}\right|_{T}=-p \delta_{i}^{j}\left(\sqrt{k^{2}+1}-\cos (k \alpha)\right)^{2} / R^{2} k^{2}$. Since $\left(\sqrt{k^{2}+1}-\cos (k \alpha)\right)^{2} / R^{2}=r^{-2}$, where $r$-distance from of torus axis to the point on the torus, then the pressure on the surface $P \propto r^{-2}$. The volume of the solid torus with main radius $c=1$ is $V(g)=2 \pi^{2} g^{2}$ where $g=a / c$ - form factor, and $a$-tube radius. Notice that $g=\sin (\theta)$ where $\theta$ - inclination of Villarceau circles to the main plane of torus symmetry, we call this angle as "stream inclination". The force of pressure $\Sigma(g)=4 \pi g A \int_{0}^{\pi}(1-g \cos (\phi))^{-1} d \phi$. Let's find the form factor $g$ when the ratio of the solid torus volume to the force of pressure on it's boundary has a maximum: maximize $(V(g) / \Sigma(g), g=0 . .1$, location $), g=(\sqrt{2})^{-1} \approx 0.7071 \ldots$ We will call the torus meeting form factor as "optimal torus". Physically, it corresponds to the minimal relative surface free energy.

Torus in the principal symmetry plane perpendicular to its axis is characterized with two structural radii $r_{m}, r_{o}$. The corresponding wave numbers are: $k_{m}=2 \pi / c(1-g), k_{o}=$ 
$2 \pi / c(1+g)$. Indices $m$-massive, and $o$-outer are used as mnemonic. Beat of oscillations is calculated as: $\cos \left(k_{m} r\right)+\cos \left(k_{o} r\right)=2 \cos \left(r\left(k_{m}+k_{o}\right) / 2\right) \cos \left(r\left(k_{m}-k_{o}\right) / 2\right)$. If half-sum or half-difference of wave numbers would be equal to wave numbers of structural radii of tori homothetic to original one, we would have the following four expressions for possible scaling factor $K_{1}=1+g, K_{2}=1-g, K_{3}=(1+g) / g, K_{4}=(1-g) / g$. If we suggest that the orbits of at least two neighbor planets have been forming due to interactions between two tori formed in nebula, the scaling relation should be taken into consideration when their interactions are described. Scaling factor $K$ would have the value similar to the ratio of the semi-major axes of neighbor planets. We call it as an orbital scaling factor. For any value of the form factor $g,\left|\ln \left(K_{1}\right)\right|<\left|\ln \left(K_{2}\right)\right|,\left|\ln \left(K_{1}\right)\right|<\left|\ln \left(K_{3}\right)\right|$, and $\left|\ln \left(K_{1}\right)\right|<\left|\ln \left(K_{4}\right)\right|$ if $g<\sqrt{2}-1$ or $g>(\sqrt{5}-1) / 2$ thereby it corresponds to the larger volume of tori intersection, and results in maximal interactions for $K_{1}$.

Arrangement of tori group results in their intersection in space, therefore, it should affect the proximity of physical characteristics of neighbor planets in a case of planetary system formation. Actually, we would like to know what is the maximal number $C$ of the planet group members able to have similar physical parameters. Let's call the indicated group as cluster, and determine the cluster's size having intersection within one torus $r_{m} K^{(C-1)}<r_{o}$. The cluster size $C=2-\ln (1-g) / \ln (1+g)$. Since $K=1+g$, the latter would be re-written as: $C=2-\ln (2-K) / \ln (K)$. First threshold corresponding to transition from the cluster size 3 to the size 4 at orbital scaling factor $K \approx 1.3894$, being in agreement with stream inclination $\theta \approx 22.917^{\circ}$.

\section{Implications}

Solar system. Orbital scaling factor $K$ for solar system $K=1.6995 \pm 0.0224$. Difference between the theoretical value and the real one does not exceed the value of standard deviation: $\Delta K=1.7071-1.6995=0.0076$. Cluster size $C=4$. Stream inclination to ecliptic $\theta_{\varepsilon}=44.4 \pm 2^{\circ}$.

Galilean moons. Orbital scaling factor for Galilean moons $K=1.6414 \pm 0.028$. Value of orbital scaling factor is less than theoretical one on a magnitude exceeding the standard deviation: $\Delta K=0.066$. Cluster size is just as in the Solar system $C=4$. Stream inclination $\theta=39.9 \pm 2.3^{\circ}$.

Saturn's satellites. The distinction of the satellite system of Saturn is the lower value of the orbital scaling factor $K=1.2949 \pm 0.00377$, and geometrical progression is clearly recognizable up to Rhea. Further analysis is possible if for the next satellite Titan we omit 2 orbits. The same situation is seen with Iapetus. The size of cluster $C=3$. Stream inclination $\theta=17.15 \pm 0.23^{\circ}$.

What is the reason for decreasing the orbital scaling factor in satellite systems of above-mentioned planets comparatively to Solar system or theoretical value? The orbital planes lie close to the equatorial plane of the central planet, and the latter has some axial tilt, thus stream inclination on the tori forming satellite system differs from $\pi / 4$ on a value of the axial tilt. When the axial tilt does not exceed $\pi / 4$, expression for orbital scaling factor is $K_{\varepsilon}=1+\sin (\pi / 4-\varepsilon)$, where $\varepsilon$ - axial tilt. Axial tilt of Jupiter $\varepsilon=3.08^{\circ}$, thus corrected values of orbital scaling factor for Galilean moons $K_{\varepsilon}=1.668$. Axial tilt of Saturn $\varepsilon=26.7^{\circ}$, therefore corrected values of orbital scaling factor for regular moons of Saturn $K_{\varepsilon}=1.314$. Corrected values well agree with actual ones. Values of stream inclination to ecliptic for Galilean moons $\theta_{\varepsilon}=\theta+\varepsilon=43 \pm 2.3^{\circ}$ and Saturn's moons $\theta_{\varepsilon}=43.88 \pm 0.23^{\circ}$. Deviation from the optimal $\pi / 4$ does not exceed $2^{\circ}$.

Neptune's satellites. Orbital scaling factor for Neptune's regular satellites $K=1.176 \pm$ 0.002. Therefore, the size of cluster, like in the Saturn's system, $C=3$. Also, like in the 
Saturn's system, two orbits between Larissa and Proteus are omitted. Omitting two orbits might reflect regularization when the size of cluster $C$ is 3 . Stream inclination to the equatorial plane $\theta=10.15 \pm 0.12^{\circ}$. Since the Neptune's axial tilt $\varepsilon=28.32^{\circ}$, the calculated value of orbital scaling factor is $K_{\varepsilon}=1.287$. Significant difference from the actual value is evident. For its analyzing, suppose that stream inclination on the torus forming Neptune's orbit differs from the optimal value $\theta_{\varepsilon}<\pi / 4$. Value of stream inclination on the torus forming the Neptune's orbit: $\theta_{\varepsilon}=\theta+\varepsilon=38.47^{\circ}$. Thereafter, ratio of Neptune's to Uranus' semi-major axes should be less than 1.707: $K=1+\sin \theta_{\varepsilon}=1.622$. Indeed, ratio of Neptune's to Uranus' semi-major axes is lowest in the family of giant planets. Taking into consideration the eccentricity of Uranus' and Neptune's orbits, the actual value of ratio of semi-major axes is $a_{N} / a_{U}=1.57 \pm 0.045$. Thus, the estimation fits well the actual value.

Uranus' satellites. Orbital scaling factor $K=1.455 \pm 0.0146$. The cluster's size $C=4$. Stream inclination to the Uranus' equatorial plane $\theta=27.07 \pm 0.95^{\circ}$. Ratio of Uranus' to Saturn's semi-major axes is largest not only in the group of giant planets, but in the Solar system in general. Subject to eccentricity, $a_{U} / a_{S}=2.01 \pm 0.1$. Orbital scaling factor is higher than maximal value 2 allowed by the model, however, there is an area of values $K \in[1.91 . .2]$ due to eccentricity of Saturn's and Uranus' orbits allowed by the model. The corresponding interval of the stream inclination to the ecliptic is $\theta_{\varepsilon} \in\left[65.6^{\circ} . .90^{\circ}\right]$. Since Uranus' axial tilt $\varepsilon=97.77^{\circ}$, stream inclination on torus forming Uranus' orbit in relation to ecliptic is $\theta_{\varepsilon}=\varepsilon-\theta=70.70^{\circ}$. Corresponding value of orbital scaling factor for Uranus vs Saturn would be $K=1+\sin \theta_{\varepsilon}=1.944$, well fitting the actual value.

Pluto's satellites. Significant gap exists between the Charon's and Nix's orbits. Presumably, this gap is caused by regularization in a cluster sized $C=3$, and two orbits are omitted. Then orbital scaling factor $K=1.351 \pm 0.003$. Stream inclination to equatorial plane is $\theta=20.55 \pm 0.18^{\circ}$. Pluto's orbit is characterized by significant inclination $i=17.14175^{\circ}$, and it should be taken into consideration while calculating the stream inclination to the ecliptic plane. Since Pluto's axial tilt $\varepsilon=119.591^{\circ}$, stream inclination in the area of Pluto's orbit $\theta_{\varepsilon}=\pi-(i+\varepsilon+\theta)=22.7^{\circ}$. Thus, ratio of semi-major axes of Pluto and Neptune $K=1.386$. Actual value is $a_{P} / a_{N}=1.313$.

Phobos and Deimos. Ratio of semi-major axes $a_{\text {Deimos }} / a_{P h o b o s}=2.50$. Let's imagine that there is lack of two orbits as it is usual for the cluster with size $C=3$. In such case, orbital scaling factor $K=\left(a_{\text {Deimos }} / a_{\text {Phobos }}\right)^{1 / 3}=1.357$, and the corresponding value of stream inclination to the equatorial plane $\theta=20.95^{\circ}$. Taking into consideration Mars' axial tilt $\varepsilon=25.19^{\circ}$, we found flow inclination to the ecliptic plane $\theta_{\varepsilon}=\theta+\varepsilon=46.14^{\circ}$ being close to the optimal value $\pi / 4$.

\section{References}

Kamchatnov, A. M. 1982, Sov. JETP, 82, No1, 117

Semenov, V. S., Korovinski, D. B., \& Biernat, H. K. 2001, preprint (arXiv: physics/0111212v1)

Arnold, V. I. \& Khesin, B. A. 1998, in Topological Methods in Hydrodynamics, (Springer-Verlag, New York)

Gil-Merdano, O. \& Llinares-Fuster, E. 2001, Math. Ann., 320, 531

Yampolsky, A. 2003, Acta Math. Hungar., 101, 73

Gibbons, G. W. 2006, Applications of Differential Geometry to Physics Part III (Cambridge University, Great Britain) 